\title{
Urgentistes au bord de la crise de nerf...
}

\author{
Emergency doctors: close to burn-out
}

\author{
F. Braun \\ C SFMU et Springer-Verlag France 2012
}

L'exercice de la médecine d'urgence cumule plusieurs facteurs de stress professionnel. Dans une publication récente, le Department of Homeland Security des États-Unis [1] les met tristement en évidence : exposition répétée à des incidents traumatisants, fatigue liée à des horaires décalés ou prolongés, déséquilibre entre les obligations professionnelles et personnelles... De nombreuses études internationales ont déjà démontré l'incidence de ces situations d'épuisement du corps médical et les urgentistes semblent y être particulièrement exposés $[2,3]$. Pourtant, peu de travaux français ont cherché à les identifier.

Dans ce numéro, Sende et al. [4] nous proposent un travail original issu d'une enquête réalisée auprès de 72 structures d'urgences françaises. Bien que cette étude descriptive présente des limites (biais de sélection par adhésion), les résultats révèlent néanmoins une situation préoccupante. Même s'ils s'estiment heureux (71\%), plus de la moitié des urgentistes de ce panel sont pourtant en situation de «pré burn-out » et $11 \%$ en état de « burn-out ». Cette enquête identifie les facteurs principaux du stress et les stratégies comportementales développées par les urgentistes pour le combattre [5]. Le burn-out marque l'étape ultime de l'épuisement. Il s'articule autour de trois dimensions [6] : épuisement émotionnel, déshumanisation de la relation d'aide et diminution de l'accomplissement personnel. Risquant d'engendrer des erreurs médicales, sa prévention devient un enjeu majeur de la gestion des risques dans les structures d'urgence.

Pour les personnes concernées, la réorientation professionnelle vers une activité plus « sereine" semble être une solution fréquente. En étudiant les urgentistes du département de la Loire, Vermare et Frappé [7] mettent en lumière ces médecins qui abandonnent la médecine d'urgence. Même si ce travail reste limité par son côté « départemental » et par un manque d'exhaustivité, il a le mérite de s'intéresser à une population médicale trop souvent malmenée, et nous

\section{F. Braun $(\square)$}

Service des urgences, Samu-Smur, CHR Metz-Thionville,

1, place Philippe de Vigneulles, F-57000 Metz, France

e-mail : fr.braun@chr-metz-thionville.fr apporte des enseignements pertinents sur les risques évolutifs de notre profession. Sur la période étudiée (2000-2010), les auteurs ont montré que 10 urgentistes sur les 43 ayant répondus $(23 \%)$ ont abandonné leur exercice professionnel dont 6 au profit de la médecine générale, spécialité d'origine de nombreux urgentistes. Les motifs de réorientation invoqués coïncident avec ceux favorisant le burn-out (survenue d'événements indésirables, violences et harcèlement, planning et temps de travail).

Ces deux articles originaux montrent bien le lien entre les difficultés actuelles de l'exercice de la médecine d'urgence et la gestion, plus ou moins efficace, des facteurs de stress par les urgentistes. Parmi les facteurs de stress identifiés dans ces travaux, certains imposent des mesures correctrices immédiates [8] :

- renforcer les effectifs lors des pics d'activité des structures d'urgence pour lutter contre les surcharges périodiques de travail, recruter des effectifs médicaux suffisants [9] ;

- mettre en place des formations spécifiques afin d'améliorer certaines compétences (pédiatrie, obstétrique...)[10] ;

- adapter les locaux des services d'urgences [11] ;

- et combattre l'inertie de l'aval (lenteur des examens complémentaires et avis spécialisés) en travaillant à l'échelle d'un établissement ou d'une région sur les protocoles de prise en charge, les filières de soins, plateaux techniques requis par pathologie...

D'autres facteurs (pression du patient et de son entourage, conflit entre collègues) nécessitent la mise en place de programmes de bien-être (wellness) et de développement de la résistance (resilience) à l'échelle individuelle comme institutionnelle [1]. Des moyens d'action existent donc [12] et doivent être mis en application.

Gardons toutefois à l'esprit que le syndrome de burn-out est un trouble d'installation progressive relevant essentiellement d'une pathologie chronique du stress, et que la souffrance des soignants face à des interventions potentiellement traumatisantes (décès d'enfants, morts violentes, multiples...) doit également être prise en compte. 


\section{Références}

1. Colvin HM, Taylor RM (2012) Building a resilient workforce. Opportunities for the Department of Homeland Security. The national academies press, Washington DC

2. Popa F, Raed A, Purcarea VL, et al (2010) Occupational burnout levels in emergency medicine-a nationwide study and analysis. J Med Life 3:207-15

3. Popa F, Arafat R, Purcarea VL, et al (2010) Occupational burnout levels in emergency medicine-a stage 2 nationwide study and analysis. J Med Life 3:449-53

4. Sende Jbeili C, Schvahn S, et al (2012) Facteurs de stress et conséquences du stress en médecine d'urgence : enquête nationale. Ann Fr Med Urg 2:(in press)

5. Hugues PH, Baldwin DC, Sheehan DV, et al (1992) Resident physician substance use by specialty. Am J Psychiatry 129:1348-54

6. Molenda S (2010) Le syndrome de burn-out. Urgences http:// www.sfmu.org/urgences2012/urgences2010/donnees/fs-recherche/fs_103_molenda inf.htm (dernier accès le 10 juin 2012)

7. Vermare E, Frappé P (2012) Abandons de carrière en médecine d'urgence. Ann Fr Med Urg 2:(in press)
8. Vermeiren E (2010) Le burn-out chez les soignants et dans les équipes. Urgences http://www.sfmu.org/urgences2012/urgences2010/donnees/fs-recherche/fs 009 vermeiren.htm (dernier accès le 10 juin 2012)

9. Samu et Urgences de France (2011) Recommandations sur les effectifs des structures d'urgence : les ressources médicales et non médicales nécessaires au bon fonctionnement des structures d'urgence. http://www.samu-de-france.fr/fr/ressources pro/exercice_pro/exercice_pro_actu/front/afficher/?id_actu $=654$ (dernier accès le 10 juin 2012)

10. Mercier JC, Martinot A, Parez N, et al (2012) Référentiel métiercompétences pour la surspécialité de médecine d'urgence pédiatrique. Ann Fr Med Urg 2:139-46

11. Société francophone de médecine d'urgence (2004) Recommandations de la SFMU sur l'architecture des services d'urgence http://www.sfmu.org/fr/ressources/referentiels/recom_sfmu_archi_service_urg.pdf (dernier accès le 11 juin 2012)

12. Schmitz GR, Clark M, Heron S, et al (2012) Strategies for coping with stress in emergency medicine: Early education is vital. J Emerg Trauma Shock 5:64-9 